the model, its advantages, and its limitations. Two issues are germane in this context.

1. PD symptoms differ fundamentally from Axis I symptoms. Axis I disorders are sometimes called "symptom disorders" because they are characterized by prominent, psychologically painful symptoms (e.g., depressed mood, difficulty sleeping, binge eating). Whether or not patients choose to acknowledge them when asked, they are typically aware of experiencing these symptoms (even if not fully aware of the symptoms' negative impact). The situation is very different for Axis II PDs, which have traditionally been conceptualized as being "ego syntonic" (i.e., consistent with the patient's experience of self). As a result, personality-disordered patients typically have far less insight into their symptoms than do patients with Axis I disorders, which complicates diagnosis, decreases motivation for treatment, and reduces therapeutic efficacy (Peters 1990; Shedler \& Westen 1999).

In the context of the network model, these Axis I-Axis II differences have two noteworthy implications. First, although self-report assessment tools (e.g., questionnaires, diagnostic interviews) are the measures of choice for rendering Axis I diagnoses, such measures are of limited value in rendering Axis II diagnoses (see, e.g., Widiger \& Samuel 2005). Indirect measures (e.g., free-response tests) and reports from knowledgeable informants must be used in conjunction with self-report instruments to assess PDs reliably (Bornstein 2007).

Second, these Axis I-Axis II differences in insight and selfawareness suggest that the definition of latent variable as conceptualized in the network model must be expanded when applied to Axis II. Here it is not only necessary to distinguish observable symptoms from unobservable latent constructs, as the network model suggests, but also to distinguish symptoms that are phenomenologically latent (i.e., ego syntonic) from those that are experienced as problematic by the patient (i.e., ego dystonic).

2. Unlike Axis I criteria, Axis II criteria are revised to minimize comorbidity. In many clinical settings the most common Axis II diagnosis is "mixed PD," and epidemiological data indicate that a sizeable proportion of PD-diagnosed patients - more than $50 \%$ in some samples - receive two or more PD diagnoses (Bornstein 2003; Widiger \& Clark 2000). As a result, symptom revision across successive editions of the DSM entails somewhat different goals on Axis I and Axis II. On Axis I symptoms are revised to increase diagnostic accuracy, but on Axis II symptoms are revised to maximize accuracy while simultaneously reducing comorbidity.

As I have noted elsewhere (Bornstein 2003), when Axis II symptoms are reworded or removed merely to limit escalating comorbidity rates, clinicians are choosing to alter reality (i.e., a high level of PD comorbidity) to fit some idealized conceptualization of PDs as distinct and separate syndromes. For example, "frantic efforts to avoid real or imagined abandonment" (see Diagnostic and Statistical Manual of Mental Disorders, 3rd edition, revised [DSM-III-R]; American Psychiatric Association 1987 , p. 347) was removed from the dependent PD criteria in $D S M-I V$ because patients with borderline PD also show this symptom, but every extant model of dependent PD would argue for inclusion of this symptom (Bornstein 2005). Removing certain PD symptoms merely to minimize PD overlap is akin to arguing that labored breathing should no longer be considered a symptom of pneumonia because patients with emphysema also show this symptom. Clearly, the contrasting strategies used to revise symptoms on Axis I and Axis II present a challenge when the network comorbidity model is extended from symptom disorders to personality pathology.

Without question, Cramer et al.'s network model represents a promising new approach to conceptualizing and quantifying comorbidity in psychiatric diagnosis. This perspective not only captures dynamic features of psychopathology that traditional latent variable models cannot capture, but has the additional advantages of shifting the focus from surface behavior to underlying process, and the level of analysis from syndrome to symptom. Extending the network model to Axis II will be challenging, but likely to benefit the model over the long term by compelling researchers to confront conceptual and empirical challenges that do not arise when the model is applied to Axis I. Questions regarding the generalizability of symptom clusters, nodes, and bridge symptoms across culture, age, and gender are almost certain to emerge, and as research on the network model advances, it will not only be useful to extend this model from Axis I to Axis II, but to begin to address aspects of crossaxis comorbidity as well.

\section{Aligning psychological assessment with psychological science}

\author{
doi:10.1017/S0140525X10000737
}

\section{Daniel Cervone}

Department of Psychology ( $m c$ 285), University of Illinois at Chicago, Chicago, IL 60607-7137.

dcervone@uic.edu

http://www.psch.uic.edu/dcervone.asp

Abstract: Network analysis is a promising step forward in efforts to align psychological assessment with explanatory theory in psychological science. The implications of Cramer et al.'s analysis are quite general. Networks analysis may illuminate functional relations not only among observable behaviors that comprise psychological disorders, but among cognitive and affective processes that causally contribute to everyday experience and action.

One of contemporary psychology's more curious features, long noted (Cervone 1991; Mischel 1973), is that the strategies through which investigators pursue two of the fields' primary goals are discordant. One goal is to understand the workings of the mind. Although there may be disagreement on the details - the precise nature of mental mechanisms and abilities, and the degree to which they are innately specified or developed through interaction with the physical and social world - there is consensus at a broad strategic level. The mind is a complex system of distinct yet functionally related parts (e.g., Barsalou 1999; McClelland \& Rogers 2003). Multiple interacting subsystems, both cognitive and affective, contribute to experience and action (Cervone 2004; Mischel \& Shoda 1995; Sander et al. 2005). "Observable behavior is an interaction effect par excellence” (Fodor 1983, p. 1).

The other goal is to assess qualities of the individual. Assessment efforts commonly describe people with respect to constructs identified in latent variable analyses. In clinical diagnosis, the constructs are diagnostic categories. In personality trait psychology, they are dimensions (Costa \& McCrae 1992). In either case, as Cramer et al. highlight, the common cause hypothesis of latent variable modeling dictates that the observable behaviors indicative of a given category or dimension are not functionally related to one another. Local independence among the indicators is assumed.

At the level of substantive theory, few psychologists are likely to embrace the common cause hypothesis and its consequences. Clinicians commonly reject essentialist views in which a diagnostic category corresponds to a singular cause (Ahn et al. 2006). Investigators who employ latent variable modeling in their research may, when pressed, abandon its common cause assumptions on theoretical grounds (see Cervone et al. 2006). "Problem and method," then, "pass one another by" (Wittgenstein 1953/ 2001, p. 197). Even investigators who recognize that actions, affects, and cognitions interact adopt latent variable methods that obscure these interactions from view.

The resulting conceptual contrast is just as sharp as Cramer et al. suggest. Consider a standard psychological science 
account of a person's tendencies to experience emotions such as anxiety and fear ("symptoms," in the case of disorders). The affective scientist might explain them by reference to interactions among physiology, cognitive appraisals processes, and enduring beliefs (e.g., Sander et al. 2005). A developmentalist would add that temperament, which itself develops partly through environmental interaction, contributes to this interacting system of cognitive and affective elements (Schmidt \& Fox 2002). Importantly, in any such account, emotional tendencies such as anxiety and fear are the explananda, the phenomena to be explained. The interacting mental systems are the explanans.

The latent variable approach turns this world upside down (Cervone 1999). In comorbidity analyses, the high-level psychological tendency "internalizing" (Krueger 1999) does explanatory work: It explains correlations among lower-level locally independent tendencies such as anxiety and fear. The tendencies to experience anxiety and fear, in turn, do more explanatory work: They explain correlations among yet lower-level locally independent tendencies such as the experience of panic and social anxiety. And so it goes; it is locally independent tendencies all the way down. Not only are there no interactions among indicators of a given construct; there also is no explanation of experience and action by reference to well-defined systems of mind or brain.

This might be palatable if investigators were careful to describe latent variables merely as clusters of interrelated behaviors. But, instead, they commonly discuss them in terms normally reserved for structural entities with causal power (Cervone 2005). For example, internalizing, a construct that summarizes between-person correlations among indices of psychological distress, is said to be a "substrate" (Krueger 1999, p. 926) of mental disorders. This is not unlike a geologist positing a substratum of "destructiveness forcefulness" to explain a region's tendency to experience both volcanoes and earthquakes.

Explanations that reference abstract tendencies of the sort identified in latent variable analyses are seductive (Kagan 1998) - so much so that, once, even Cramer et al. are enticed. Did they really mean to say that "neuroticism" - a latent variable that reflects intercorrelations among dispositional tendencies to experience anxiety, hostility, self-consciousness, impulsiveness, vulnerability, and depression (Costa \& McCrae 1992) - "can trigger the onset of depression" (sect. 4, para. 5, emphasis added)? Neuroticism is ripe for network analysis. One would not claim that it "triggers" depression, since depression is part of neuroticism, and neuroticism is conceptualized as a constant (see Borsboom et al. 2003).

The great virtue of Cramer et al.'s article is that they not only articulate a problem, but provide a solution: network analysis. Future work might expand their current scope. In principle, networks could include functional relations not only among observable behaviors, but among cognitive and affective components as well; empirical evidence documents numerous functional relations, such as the influence of selfconsciousness on emotion (Mor \& Winquist 2002), self-efficacy perceptions on motivation (Bandura 1997), knowledge structures on self-appraisals (Cervone et al. 2008), and mood on self-evaluations (Cervone et al. 1994). One might account for personality traits in the manner Cramer et al. account for diagnostic categories and comorbidities. Functional relations among perceived self-efficacy, personal goal-setting, and disciplined, persistent behavior (e.g., Bandura \& Cervone 1986), for example, might enable one to view conscientiousness (whose components include competence, achievement striving, self-discipline, and dutifulness; Costa \& McCrae 1992) as a cluster of functionally interrelated cognitive-affect elements and their behavioral effects.

Such an effort requires an assessment method that taps these cognitive and affective elements. A social-cognitive approach to assessment (Cervone et al. 2001) is apt in that it addresses "functional relations among affect and physiological arousal, cognition, and action" (Cervone et al. 2001, p. 41) rather than latent variables measured by independent indicators. Social-cognitive methods, and recent clinical assessment efforts (Haynes et al. 2009), are sensitive to individual idiosyncrasy, thus addressing Cramer et al.'s recognition of possible individual-level variability in network structure.

For more than four decades, psychologists have called for assessment and measurement strategies that align with the body of knowledge available in psychological science (Mischel 1968). Cramer et al.'s contribution is a most valuable step in this direction.

\section{ACKNOWLEDGMENTS}

I thank Tracy L. Caldwell and Yuichi Shoda for their comments on a draft of this commentary.

\section{Comorbid science $?^{1}$}

\section{doi:10.1017/S0140525X10000609}

\section{David Danks, ${ }^{\mathrm{a}, \mathrm{b}}$ Stephen Fancsali, ${ }^{\text {a }}$ Clark Glymour, ${ }^{\mathrm{a}, \mathrm{b}}$ and Richard Scheines ${ }^{a}$ \\ ${ }^{a}$ Department of Philosophy, Carnegie Mellon University, Pittsburgh, PA 15213, and ${ }^{\mathrm{b}}$ Institute for Human and Machine Cognition, Pittsburgh, PA 15213. \\ ddanks@cmu.edu \\ http://www.hss.cmu.edu/philosophy/faculty-danks.php \\ sfancsal@andrew.cmu.edu \\ cg09@andrew.cmu.edu \\ http://www.hss.cmu.edu/philosophy/faculty-glymour.php \\ scheines@cmu.edu \\ http://www.hss.cmu.edu/philosophy/faculty-scheines.php}

Abstract: We agree with Cramer et al.'s goal of the discovery of causal relationships, but we argue that the authors' characterization of latent variable models (as deployed for such purposes) overlooks a wealth of extant possibilities. We provide a preliminary analysis of their data, using existing algorithms for causal inference and for the specification of latent variable models.

We agree with the view that Cramer et al. develop in the target article: that naïve latent variable models often fall woefully short of ideal. Unfortunately, their proposed solution and accompanying test case suffer from a number of flaws.

Cramer et al. begin with a straw man: They assume that, in a latent variable model, symptoms cannot also influence one another. Unless we define "latent variable model" to exclude such effects, there is no reason to impose such a constraint on our models. Mathematically, it is straightforward for latent variable models to have both latent common causes of measured variables and direct influences of measured variables on other measured variables. This is often the case for actual causal structures; for example, when there is confounding in observational or quasi-experimental studies.

Cramer et al. further claim that a "latent variable model renders all symptoms equally central and thus exchangeable" (sect. 5, para. 3). This claim is difficult to understand. "Central" is neither a causal nor a statistical notion; "exchangeable" is a statistical notion that, if meant, would be quite inappropriate in this usage. Cramer et al. might mean that in latent variable models all symptoms have the same variance, or the same dependence on any latent variables, or in their probability distributions conditional on values of latent variables, or in their probabilities conditional on one another. Each of these claims is violated in many latent variable models in the social sciences and elsewhere, and all of these claims are false unless "latent variable model" is arbitrarily defined so as to satisfy them. But that would be to focus on 